\section{Meal frequency and duration of overnight fast: a role in gall-stone formation?}

Supersaturation of bile with cholesterol is a necessary condition for the formation of cholesterol gall stones. ${ }^{1}$ Most normal people have lithogenic bile after an overnight fast, ${ }^{2}$ and short-term fasting is associated with a decreased rate of biliary bile-acid secretion and an increase in cholesterol saturation of hepatic and gall-bladder bile. ${ }^{3-5}$ Thus meal frequency - that is, the duration of fasting throughout the day-might have an effect on gall-stone formation. We therefore tested this hypothesis by comparing, in a prospective case-controlled study, the duration of overnight fasting in patients with gall stones and controls.

\section{Patients, methods, and results}

We studied 380 consecutive women outpatients referred to the department of radiology for oral cholecystography. The main indications for this investigation were pain in the right hypochondrium, dyspepsia, and migraine. The patients were divided into two groups: 115 with radiolucent gall stones and 245 with a normal gall bladder, who served as controls. The control patients were individually matched to patients with gall stones for age (within five years), body weight $(5 \mathrm{~kg}$ ), number of pregnancies, and consumption of lithogenic drugs (oral contraceptives, clofibrate). The final study population consisted of 47 case-control pairs. The mean age and weight of the patients with gall stones and the controls was $50 \pm S D 16$ years and $61 \pm 10 \mathrm{~kg}$, respectively.

The duration of fasting was measured from the end of the last meal of the day (always dinner) until the beginning of the next meal (breakfast or lunch). The precise composition of the breakfast was determined in all subjects. Drinking, on waking, only cup(s) of black or white coffee with or without sugar was considered to be similar to a total fast. We had verified in 10 women volunteers, by real-time ultrasonography, that coffee did not cuuse gall-bladder emptying. Statistical analysis was carried out using the Wilcoxon signed-rank test for paired samples.

Total fast in the morning was noted in 11 patients with gall stones and five controls. The duration of overnight fasting (mean + SEM) in the whole group was not significantly different between the patients with gall stones $(13 \mathrm{~h} 30 \mathrm{~min}+36 \mathrm{~min})$ and the controls $(12 \mathrm{~h} 47 \mathrm{~min} 18 \mathrm{~min})$. In the youngest patients (20-35 years), however, total fast was noted in six out of 12 patients and two out of 12 controls. In these young subjects duration of overnight fasting was significantly longer in the patients with gall stones $(14 \mathrm{~h} 41 \mathrm{~min} \pm 48 \mathrm{~min})$ than in the controls $(12 \mathrm{~h} 41 \mathrm{~min} \pm 39 \mathrm{~min} ; \mathrm{p}<0.05)$. We verified by using covariance analysis that no other factor modified the interpretation of this result-ior example, mean body weight in the young patients with gall stones and the controls was $60 \cdot 8+8 \cdot 8 \mathrm{~kg}$ and $60 \cdot 2+9 \cdot 2 \mathrm{~kg}$, respectively.

\section{Comment}

This study shows that the overnight fast-that is, the interval between dinner and the following meal, be it breakfast or lunch-is significantly grater in young women with gall stones (20-35 years) than in women in the same age range without gall stones. As the patients and their controls were closely matched for the well-known risk factors of cholesterol cholelithiasis these results suggest that reduction in meal frequency and prolongation of overnight fasting might increase the chances of gall-stone formation at least in younger people. In the older age groups this time difference was not found; it is difficult, however, to exclude the possibility that these patients, when younger, had had different dietary habits. In this north-western region of France the times of lunch and dinner invariably ranged from 12 noon to $1 \mathrm{pm}$ and from 7 to $8 \mathrm{pm}$, respectively. By contrast, there are large individual variations in the existence, time, and composition of breakfast. In young people breakfast is often not eaten or limited to coffee, but presumably it becomes more regular and substantial with aging.

These results agree with several studies showing an increase in the cholesterol saturation of hepatic and gall-bladder human bile after an eight- to 16-hour fast. ${ }^{3-5}$ Fasting is associated with the storage of a part of the bile-acid pool in the gall bladder, a decrease in the rate of biliary bile-acid secretion, without a parallel diminution in the biliary secretion of cholesterol, and the hepatic production and secretion of supersaturated bile. This effect might be accentuated by previous bileacid deficiency or excess cholesterol secretion or both. Finally, if mixing of supersaturated bile in the gall bladder is inadequate or incomplete cholesterol precipitation and crystal growth might occur in localised regions of the gall bladder and promote gall-stone formation. ${ }^{3}$

We conclude that, at least in young women, reduced meal frequency and short-term prolongation of fasting might increase the risk of gallstone formation.

1 Small DM. Cholelithiasis. Pathogenesis of cholesterol gall-stone disease. In: Bockus HL, ed. Gastroenterology. Vol 3. Philadelphia: Saunders, 1976:740-52.

${ }^{2}$ Holzbach RT, Marsh M, Olszewski M. Cholesterol solubility in bile. Evidence that supersaturated bile is frequent in healthy man. $\mathcal{f}$ Clin Invest 1973;25:1467-79.

${ }^{3}$ Metzger AL, Alder R, Heymsfield S, Grundy SM. Diurnal variation in biliary lipid composition. Possible role in cholesterol gall-stone formation. N Engl f Med 1973;288:333-6.

${ }^{4}$ Williams NC, Morse JWI, MacDonald IA, Kotoor R, Riding MD. Increased lithogenicity of bile on fasting in normal subjects. Am $\mathcal{F}$ Dig Dis $1977 ; 22: 189-94$.

${ }^{5}$ Bloch HM, Thornton JR, Heaton KW. Effects of fasting on the composition of gallbladder bile. Gut 1980;21:1087-9.

(Accepted 5 August 1981)

Clinique Médicale A, Centre Hospitalier Universitaire, 80030 Amiens, France

J P CAPRON, MD, physician

J DELAMARRE, MD, physicia

M A HERVE, MD, physician

J L DUPAS, MD, physician

$P$ POULAIN, MD, physician

$P$ DESCOMBES, MD, physician

\section{Antihistamine treatment: a patient self-assessment method in chronic urticaria}

Chronic urticaria is common and intractable. Occasionally an underlying cause is identifiable, but most patients have to await eventual spontaneous remission of this distressing disorder. Systemic antihistamines give some symptomatic relief, though their capricious efficacy and troublesome side effects make them unsatisfactory to both patients and doctors. A current commercial index of proprietary preparations lists 20 separate antihistamines, which reflects the inadequacy of this class of drug. Since people's responses to particular antihistamines vary considerably ${ }^{1}$ it is crucial to select the drug best suited to a given patient. A self-assessment questionnaire used in patients with rheumatoid arthritis receiving non-steroidal antiinflammatory drugs has been described, ${ }^{2}$ though no data on its value in practice were given. This method has now been modified for use in patients with chronic idiopathic urticaria who require antihistamine treatment, and I report here results obtained in 18 patients.

\section{Patients, methods, and results}

Eighteen adults attending the outpatient department of St John's Hospital for Diseases of the Skin were studied. Chronic idiopathic urticaria was defined as the occurrence of extensive wealing daily for at least three months. Pre-existing drug treatment was withdrawn for at least three days before the start of the study.

Self-assessment questionnaire-Each patient was given a self-assessment questionnaire (figure). Six antihistamines were given in random order, but no attempt was made to carry out the study blind. In a preliminary study a placebo was included: since this had no detectable influence on the severity of the urticaria and was not associated with side effects its use was discontinued. Each drug was taken by number for five days beginning on Monday and ending on Friday. No treatment was given on Saturday or Sunday, to prevent overlap between successive drugs. Patients were warned both verbally and on the questionnaire of the dangers of alcohol consumption while receiving antihistamine treatment, and possible relevant occupational hazards were also discussed. At the end of each treatment day the patient recorded the following information: approximate number of weals; severity 
of itch; severity of side effects; and whether the antihistamine suited him. After completing the questionnaire the patient returned to the outpatient department for evaluation of the results. Antihistamines that the patient considered to be unsuitable were rejected. The remainder were scored for number of weals, severity of itch, and side effects. The lowest-scoring antihistamine was judged the most appropriate for the patient.

Antihistamines-At the pharmacy the patients were given six containers, each containing five days' supply of one of the following antihistamines with appropriate instructions, as detailed on the sample questionnaire: promethazine, chlorpheniramine, hydroxyzine, cyproheptadine, trimeprazine, and mepyramine.

All 18 patients successfully completed the questionnaire. Of the regimens studied, promethazine was preferred (lowest scoring) in six cases and chlorpheniramine in another six. Cyproheptadine and mepyramine each received the lowest score in two. Hydroxyzine and trimeprazine each received the lowest score in one. In instances in which two or more regimens received similar ratings, the final choice was based on simplicity and convenience. The questionnaire was filed in the patient's case notes so that the information recorded could be used to substitute an alternative low-scoring antihistamine at a future date when appropriate.

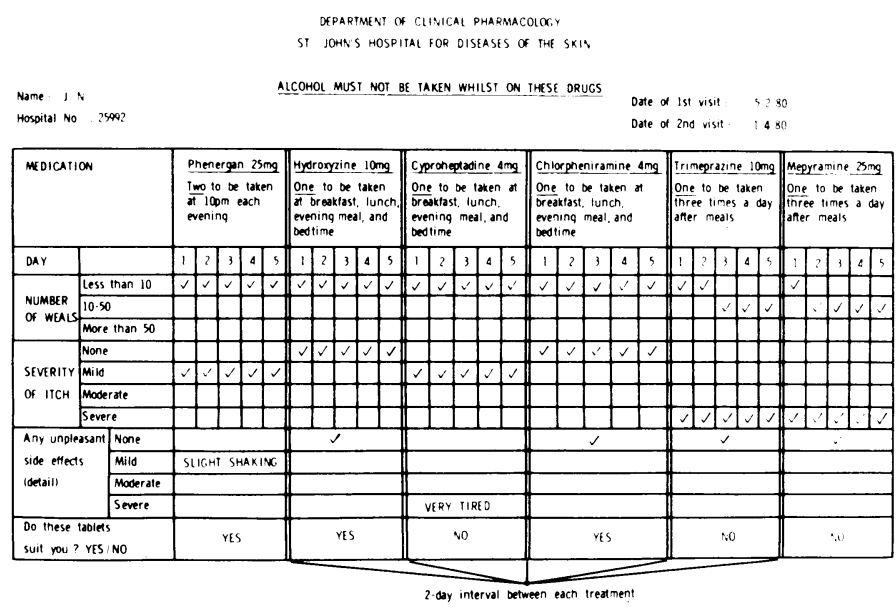

Antihistamine self-assessment questionnaire.

\section{Comment}

The self-assessment technique has proved valuable in the drug treatment of patients with chronic idiopathic urticaria. Other data, to be published elsewhere, suggest that the method is equally effective in the physical urticarias. Apart from the major advantage that antihistamine treatment is placed on a rational basis, patients appreciate active participation in their management and acquire a better insight into the nature of their skin disorder and its treatment.

This study was not intended to yield any overall conclusion about the ranking of antihistamines in order of effectiveness in patients with chronic urticaria, though the self-assessment method, with considerable design modifications, could be used for this purpose. Nevertheless, it is interesting that few patients preferred hydroxyzine and cyproheptadine, currently fashionable antihistamines in dermatological practices. Promethazine, given as a late evening dose, scored well because of a low incidence of side effects during the succeeding day and because patients found it reasonably effective. The dosage regimens chosen in this study may differ from those in routine use in other centres. The method, however, is easily adapted to accommodate individual foibles of prescribing or changing fashions in antihistamine treatment. This self-assessment method should be extended to other pruritic disorders for which antihistamines are regularly prescribed including atopic eczema.

I thank the staff of the pharmacy department, St John's Hospital for Diseases of the Skin, for their help with this study, and Dr Andrew Herxheimer for helpful comments on the manuscript.

${ }^{1}$ Melville KI. Antihistamine drugs. In: Schacter, M. ed. International encyclopedia of pharmacology and therapeutics. Oxford: Pergamon Press, 1973:127-71.

${ }^{2}$ Gumpel JM. Which anti-inflammatory drugs in rheumatoid arthritis? Br Med F 1978;ii :256.

(Accepted 21 August 1981)

Institute of Dermatology, London E9 6BX

$M$ W GREAVES, MD, FRCP, professor of dermatology

\section{Thrombosis after venography in familial antithrombin III deficiency}

We report the occurrence of venous thrombosis after venography on two occasions in a patient with familial antithrombin III deficiency.

\section{Case report}

A 25-year-old man broke his right ankle and three weeks after the application of a below-knee plaster cast presented with pain in the fractured leg. Deep venous thrombosis was suspected, but venography with $100 \mathrm{ml}$ Conray $280(60 \%$ meglumine iothalamate; equivalent $280 \mathrm{~g}$ iodine/l) flushed through with saline not containing heparin did not confirm this. Over the next few days the pain and swelling increased and a repeat venogram disclosed long lengths of fresh clot in the deep veins of the calf and almost complete occlusion of the popliteal and femoral veins. He was treated with heparin infusion and warfarin but seven days later suffered a pulmonary embolus. Familial antithrombin III deficiency was subsequently diagnosed in this unit, antithrombin III concentrations being $49 \%$ and $47 \%$ on immunological and functional assays, respectively. ${ }^{1}$

One year later the patient developed pain in the left calf and knee. Venography of this leg using $50 \mathrm{ml}$ Conray 280 flushed through with $100 \mathrm{~m}$ isotonic saline without the addition of heparin showed no thrombus. The pain in the calf became more severe, and tenderness and swelling developed. Platelets labelled with ${ }^{111}$ indium $^{2}$ clearly showed thrombus in the left calf two days later (figure).

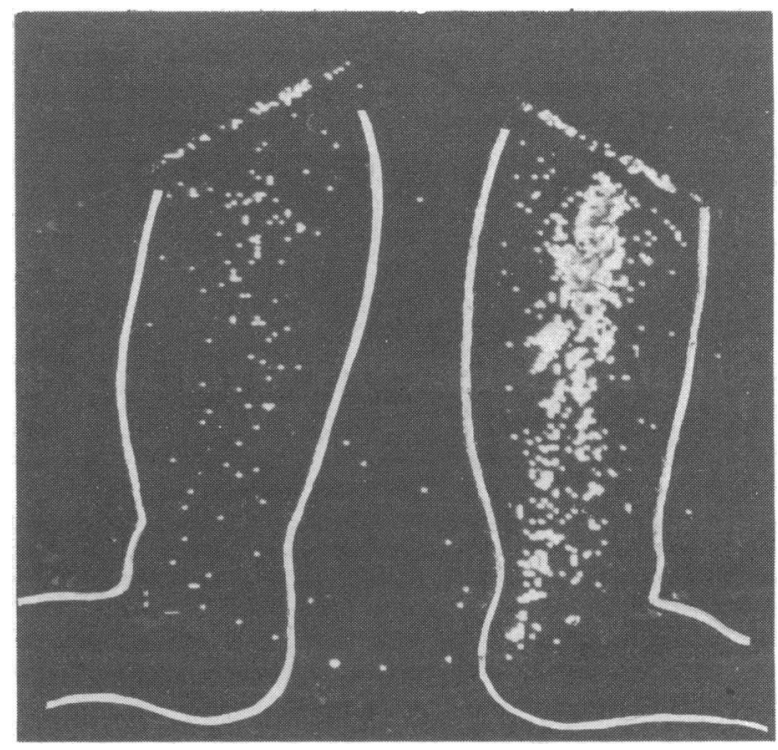

Gammascan showing massive uptake of indium-labelled platelets in upper left calf with lesser uptake along veins in lower calf, indicating damage to venous endothelium by venographic contrast medium.

\section{Comment}

Venous thrombosis may occur as a complication of venography ${ }^{3}$ but has not been described in a patient with familial antithrombin III deficiency. Patients with this disorder show a definite predisposition to venous thrombosis, which is commonly precipitated by a thrombogenic stimulus such as pregnancy or operation. ${ }^{4}$ Since venography is a recognised thrombogenic stimulus it might be expected to result in venous thrombosis in patients with antithrombin III deficiency. Our experience supports this and suggests that in these patients venography should be avoided when possible. When reliable alternative techniques for the diagnosis of deep venous thrombosis such as indium-labelled platelets are not available, it may be advisable to use metrizamide as the venographic contrast medium, since this compound appears to be associated with a low incidence of thrombotic complications, ${ }^{5}$ and to flush through the contrast medium with saline containing small quantities of heparin.

1 Winter JH, Fenech A, Bennett B, Douglas AS. Transfusion studies in patients with familial antithrombin III deficiency. $\mathrm{Br} \mathcal{F}$ Haematol (in press). $\overrightarrow{0}$ 